\section{Spontaneous decapitation of a small colorectal cancer: follow-up of the spontaneous course}
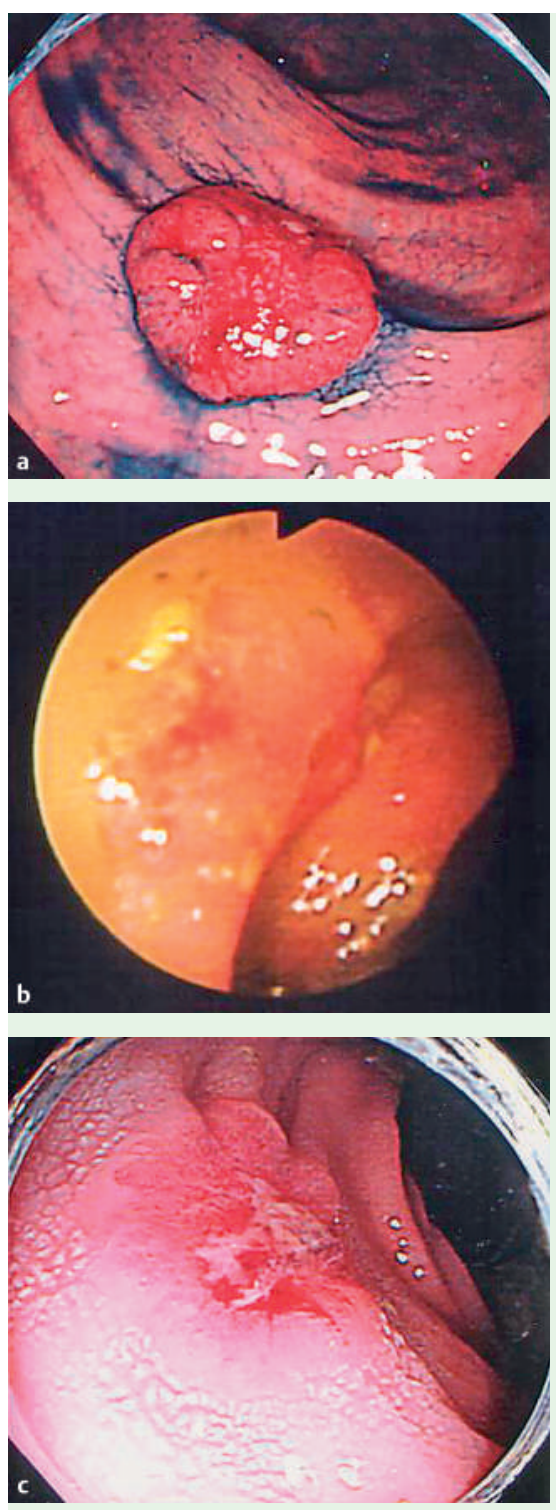

Fig. 1 Endoscopic observation of the regrowth of colorectal carcinoma. a On 15 September 2000, an elevated tumor measuring $20 \mathrm{~mm}$ was found in the rectum. b On 5 December 2000, most of the tumor had dropped off. c On 11 March 2003, the partially dislodged tumor re-
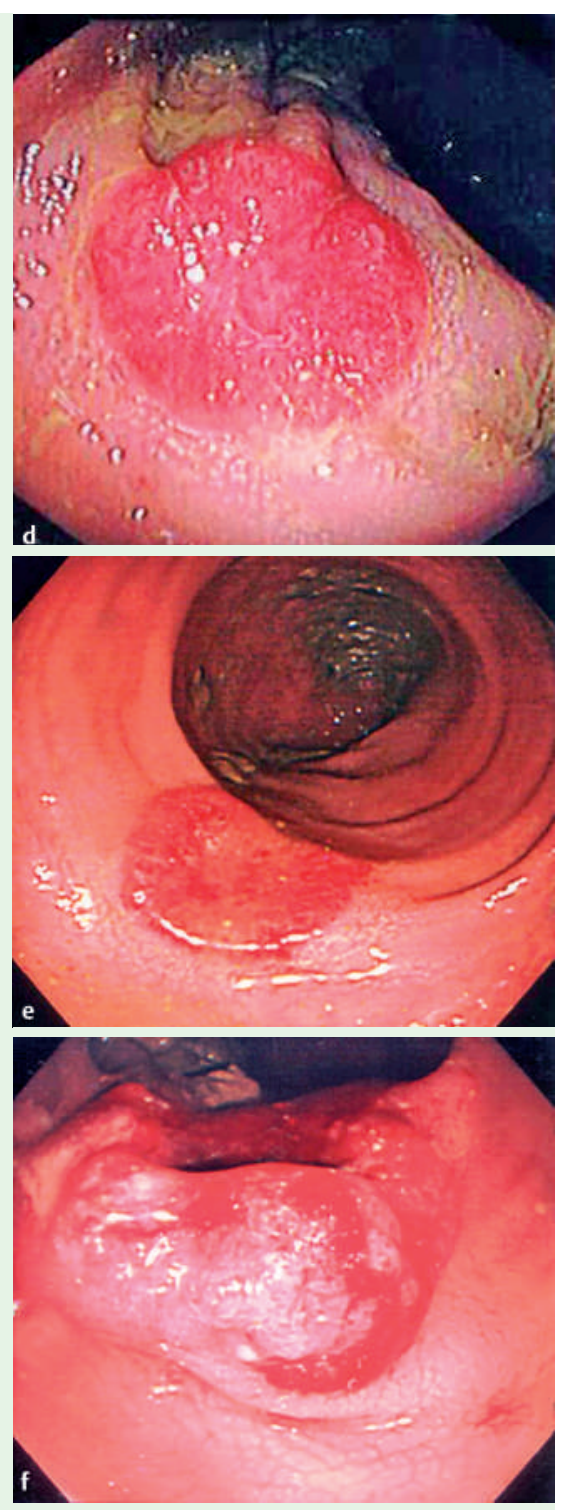

mained as a flat lesion. d On 11 November 2003 , the tumor had increased in volume and redness. e On 22 June 2004, the tumor had developed to a state approaching that observed at the first examination. f On 2 May 2005, the tumor had developed to an invasive ulcerated cancer.
An 80-year-old woman with a history of diabetes and arrhythmia underwent colonoscopy on 28 August 2000, because of a positive fecal occult blood test. An elevated tumor measuring $20 \mathrm{~mm}$ was found in the rectum ( $\bullet$ Fig. 1 a). Biopsy of three different sites revealed well differentiated adenocarcinoma.

Subsequently, on 15 September she suffered a cerebral infarction, which resulted in paralysis of the left side and aphasia. Informed consent to cancel surgery and transfer the patient to a local rehabilitation hospital was obtained from the family.

Colonoscopy was repeated on 5 December 2000 at the bedside, to re-evaluate the state of the tumor before hospital transfer. Endoscopy showed that most of the tumor had dropped off ( $\bullet$ Fig. 1 b).

On 11 March 2003, a request was received from the hospital to which she had been transferred to investigate a suspected intestinal obstruction. Colonoscopic examination revealed that the partially dislodged tumor remained as a flat lesion (ه Fig. $1 \mathrm{c}$ ). Impaired gastrointestinal transit was due to constipation. Repeat examination was scheduled for 6 months later.

On 11 November 2003, the tumor had increased in volume and redness, and biopsy showed well differentiated adenocarcinoma ( $\bullet$ Fig. $1 \mathrm{~d}$ ). The family declined active treatment and the clinical course was observed.

On 22 June 2004, the tumor had developed to a state approaching that observed during the first examination ( $\bullet$ Fig. 1 e), but the family continued to decline surgery.

On 2 May 2005, the tumor had developed to an invasive ulcerated cancer ( Fig. 1 f), and multiple liver metastases were observed on abdominal computed tomography scan.

On 20 November 2005, the patient passed away, 1908 days after initial detection of the tumor.

This was a rare case in which the process of regrowth of colorectal carcinoma was followed by endoscopic observation, after the tumor had dropped off due to the mechanical stimulation of biopsy [1].

Endoscopy_UCTN_Code_CCL_1AD_2AB 
Y. Tomiki ${ }^{1}$, H. Gonda ${ }^{2}$, E. Seki²,

T. Maeda', D. Kitamura ${ }^{2}$

${ }^{1}$ Department of Coloproctological Surgery, Juntendo University School of Medicine, Tokyo, Japan

2 Department of Surgery, Ohta General Hospital, Kawasaki, Japan

\section{References}

1 Paul RE Jr, Gherardi GJ, Miller HH. Autoamputation of benign and malignant colonic polyps: report of two cases. Dis Colon Rectum 1974; 17: 331 - 335

\section{Bibliography}

DOI 10.1055/s-2007-966729

Endoscopy 2007; 39: E290 -E291

(c) Georg Thieme Verlag KG Stuttgart · New York . ISSN 0013-726X
Corresponding author

\section{Y. Tomiki, MD}

Department of Coloproctological Surgery Juntendo University School of Medicine 2-1-1 Hongo Bunkyo-ku

Tokyo 113-8421

Japan

Fax: +81-3-3813-0731

tomiki@muh.biglobe.ne.jp 